\title{
BTB-BACK-TAZ domain protein MdBT2-mediated MdMYB73 ubiquitination negatively regulates malate accumulation and vacuolar acidification in apple
}

Quan-Yan Zhang ${ }^{1}$, Kai-Di Gu', Jia-Hui Wang ${ }^{1}$, Jian-Qiang Yu', Xiao-Fei Wang ${ }^{1}$, Shuai Zhang $\mathbb{0}^{2}$, Chun-Xiang You', Da-Gang Hu iD ${ }^{1}$ and Yu-Jin Hao'

\begin{abstract}
As an important primary metabolite, malate plays a key role in regulating osmotic pressure, $\mathrm{pH}$ homeostasis, stress tolerance, and fruit quality of apple. The R2R3-MYB transcription factor (TF) MdMYB73 was identified as a protein that plays a critical role in determining malate accumulation and vacuolar acidification by directly regulating the transcription of aluminum-activated malate transporter 9 (MdALMT9), vacuolar ATPase subunit A (MdVHA-A), and vacuolar pyrophosphatase 1 (MdVHP1) in apple. In addition, the bHLH TF MdCIbHLH1 interacts with MdMYB73 and enhances the transcriptional activity of MdMYB73. Our previous studies demonstrated that the BTB-BACK-TAZ domain protein MdBT2 can degrade MdClbHLH1 to influence malate accumulation and vacuolar acidification. However, the potential upstream regulators of MdMYB73 are currently unknown. In this study, we found that MdBT2 directly interacts with and degrades MdMYB73 through the ubiquitin/26S proteasome pathway to regulate malate accumulation and vacuolar acidification. A series of functional assays with apple calli and fruit showed that MdBT2 controls malate accumulation and vacuolar acidification in an MdMYB73-dependent manner. Overall, our findings shed light on the mechanism by which the BTB-BACK-TAZ domain protein MdBT2 regulates malate accumulation and vacuolar acidification by targeting MdMYB73 and MdClbHLH1 for ubiquitination in apple. This information may help guide traditional breeding programs and fruit tree molecular breeding, and lead to improvements in fruit quality and stress tolerance.
\end{abstract}

\section{Introduction}

Malate, a major intermediate in several metabolic processes, is present in most plant species and plays critical roles in regulating osmotic pressure, $\mathrm{pH}$ homeostasis, nutrient absorption, and stress resistance ${ }^{1,2}$. Malate also acts as an important acidity indicator for fruit quality that

\footnotetext{
Correspondence: Da-Gang Hu (fap_296566@163.com) or Yu-Jin Hao (haoyujin@sdau.edu.cn)

${ }^{1}$ National Key Laboratory of Crop Biology, Shandong Collaborative Innovation Center of Fruit \& Vegetable Quality and Efficient Production, College of Horticulture Science and Engineering, Shandong Agricultural University, Tai-An, Shandong 271018, China

${ }^{2}$ College of Chemistry and Material Science, Shandong Agricultural University, Tai-An, Shandong 271018, China
}

influences the perception of its sweetness, the accumulation of other nutrients, and the processing quality of fruit wine and juice ${ }^{3-7}$. Malate metabolism is a complex biological process and is comprised of synthesis, degradation, and transport ${ }^{7,8}$.

Malate is synthesized during cytoplasmic glycolysis, the mitochondrial tricarboxylic acid cycle, the chloroplast Calvin cycle, and the glycosome glyoxylate cycle ${ }^{9-11}$. Malate transport involves vacuolar proton pumps, and malate transporters play key roles in malate transport from the cytosol into the vacuole, thus driving vacuolar acidification ${ }^{2}$. Vacuolar proton pumps contain vacuolar $\mathrm{H}^{+}$-ATPase $(\mathrm{V}$ ATPase) and $\mathrm{H}^{+}$-pyrophosphatase (V-PPase $)^{12}$. V-ATPase

\section{(c) The Author(s) 2020}

(c) (i) Open Access This article is licensed under a Creative Commons Attribution 4.0 International License, which permits use, sharing, adaptation, distribution and reproduction cc. in any medium or format, as long as you give appropriate credit to the original author(s) and the source, provide a link to the Creative Commons license, and indicate if changes were made. The images or other third party material in this article are included in the article's Creative Commons license, unless indicated otherwise in a credit line to the material. If material is not included in the article's Creative Commons license and your intended use is not permitted by statutory regulation or exceeds the permitted use, you will need to obtain permission directly from the copyright holder. To view a copy of this license, visit http://creativecommons.org/licenses/by/4.0/. 
has a complex structure that includes two functional parts: the peripheral $V_{1}$ sector and the membrane-embedded $V_{0}$ sector $^{13,14} \cdot V_{1}$ has eight different subunits, with $A$ and $B$ subunits critical for the catalytic activity, and $\mathrm{C}-\mathrm{H}$ subunits linked to hydrophobic and membrane-embedded $\mathrm{V}_{0}$, as is critical for ATP hydrolysis ${ }^{12,15}$. The $\mathrm{V}_{0}$ sector contains six subunits (VHA-a, -c, - $c^{\prime},-c^{\prime \prime}$, -d, and -e) that form a protonconducting channel through which protons are translocated $^{12,16}$. V-PPase, on the other hand, is composed of a single polypeptide ${ }^{17,18}$. V-ATPase and V-PPase are both necessary not only for forming and maintaining the electrochemical potential gradient on the tonoplast ${ }^{2,19}$, but also for supplying energy to other transport proteins, such as the malate transporters tDT, Ma1, and Ma10 (refs. ${ }^{7,12,20-22}$ ).

Transcription factors (TFs) are widely involved in the regulation of malate synthesis and transport. For example, SIWRKY42 directly regulates SIALMT9, negatively affecting malate concentration in tomato ${ }^{5}$. In apple, allelic variations in the MYB TF $M d M Y B 44$ impact malate concentration $^{23}$. In addition, MdMYB1 controls malate accumulation and vacuolar acidification by directly regulating the transcription of $M d V H A-B s, M d V H A-E 2$, and $M d t D T^{12}$. MdMYB73 is involved in malate accumulation and vacuolar acidification by directly regulating the transcription of MdVHA-A, MdVHP1, and MdALMT9 (ref. ${ }^{2}$ ). MdCIbHLH1, a bHLH TF, interacts with MdMYB73 and enhances its effects on targets to affect malate concentration and vacuolar acidification ${ }^{2,24}$. Studies focused on the transcriptional regulation of malate have been carried out in apples, but the effects of posttranslational regulation on malate accumulation and vacuolar acidification are still poorly understood.

BT2 is a BTB protein that functions in the regulation of telomerase in Arabidopsis ${ }^{25}$. As a cytoskeletal protein, BT2 interacts with the cullin scaffolding protein CUL3 and the RING finger protein RBX1 to form E3 ubiquitin ligase complexes ${ }^{26,27}$. Recent studies have shown that BT2 is a central component of an interconnected signaling network that detects and responds to multiple inputs, such as light, temperature, carbon and nitrogen levels, hormones, and stresses ${ }^{28-31}$. Recently, we found that the ubiquitination-related scaffold protein MdBT2 can regulate the stability of MdCIbHLH1 via ubiquitination in response to nitrate, which in turn transcriptionally reduces the expression of malate-associated genes, thereby controlling malate accumulation and vacuolar acidification in apple ${ }^{24}$. In contrast to MdCIbHLH1, MdMYB73 can directly regulate the transcription of malate-related genes to adjust malate accumulation and vacuolar acidification $^{2}$. However, the potential regulators of MdMYB73 during malate accumulation are currently unknown.

In this work, we found that MdBT2 is a possible MdMYB73 interactor by employing a yeast two-hybrid $(\mathrm{Y} 2 \mathrm{H})$-based screening library. We determined that this interaction represented another ubiquitination mechanism affecting malate accumulation, in addition to the ubiquitination of MdCIbHLH1 by MdBT2. Subsequently, we discovered that MdBT2 regulates the stability of MdMYB73 via ubiquitination, which in turn transcriptionally reduces the expression of malateassociated genes, thereby altering malate accumulation and vacuolar acidification in apple. Finally, we integrated these findings into a working model to explain how MdBT2 regulates malate accumulation and vacuolar acidification in apple.

\section{Materials and methods \\ Plant materials and growth conditions}

The apple calli used in this study were induced from young embryos of the "Orin" apple (Malus domestica Borkh) ${ }^{32}$. "Orin" apple calli were grown on Murashige-Skoog medium with $1.5 \mathrm{mg} \mathrm{L}^{-1}$ 2,4-dichlorophenoxyacetic acid (2,4-D) and $0.4 \mathrm{mg} \mathrm{L}^{-1}$ 6-benzylaminopurine $(6-\mathrm{BA})$ at $25^{\circ} \mathrm{C}$ in the dark $^{33}$. These calli were subcultured three times at 15-day intervals before being used for further study ${ }^{34}$.

\section{Construction of expression vectors and genetic transformation}

The MdBT2-antisense (35S::anti-MdBT2), MdBT2-ovx (35S::MdBT2-MYC), and MdMYB73-ovx (35S::MdMYB73GFP and 35S::MdMYB73-MYC) vectors were generated using pCXSN, pCAMBIA1301, pCXCN-GFP, and pCAMBIA1301 vectors, respectively ${ }^{2,35,36}$. The partial 5'-UTR sequences of antisense $M d M Y B 73$ were also inserted into a pCXCN-GFP vector to generate antisense suppression vectors (35S::anti-MdMYB73). The accession numbers of MdBT2 and MdMYB73 in the GDR data libraries are MDP0000643281 and MDP0000894463, respectively. These constructs were introduced into "Orin" apple calli by Agrobacterium-mediated transformation using Agrobacterium tumefaciens strain LBA4404 (ref. ${ }^{37}$ ). Transgenic apple calli were selected on medium that contained $300 \mathrm{mg} \mathrm{L}^{-1}$ cephalosporin and $30 \mathrm{mg} \mathrm{L}^{-1}$ hygromycin. The expression level of $M d M Y B 73$ was determined by quantitative RT-PCR $(\mathrm{qRT}-\mathrm{PCR})^{38}$.

\section{$\mathrm{Y} 2 \mathrm{H}$ assays}

$\mathrm{Y} 2 \mathrm{H}$ assays were used to verify the interaction between MdBT2 and MdMYB73. The full-length and truncated cDNAs of MdMYB73 (amino acids 1-242, 1-80, 1-131, 81-131, and 132-242) were inserted into pGAD424, while $M d B T 2$ cDNAs and truncated sequences (amino acids 1-335, 1-195, 1-128, 129-195, 129-335, and 196-335) were inserted into pGBT9. The pGAD424MdMYB73 and pGBT9-MdBT2 plasmids were transferred into the $\mathrm{Y} 2 \mathrm{H}$ Gold strain and plated on medium lacking Trp and Leu (-T/-L) at $28^{\circ} \mathrm{C}$ for 2 days. The colonies were then transferred to medium lacking Trp, 
Leu, Ade, and His $(-\mathrm{T} /-\mathrm{L} /-\mathrm{A} /-\mathrm{H})$ and cultured for the interaction assays ${ }^{24}$.

\section{Pull-down assays}

The full-length cDNAs of MdBT2 and MdMYB73 were inserted into pGEX-4T or pET-32a, and subsequently expressed in Escherichia coli BL21 (DE3) to generate recombinant proteins MdBT2-GST and MdMYB73-HIS, respectively. The fusion proteins of MdBT2-GST, MdMYB73-HIS, and GST were used for pull-down assays with anti-HIS and anti-GST antibodies ${ }^{38}$.

\section{Bimolecular fluorescence complementation assays}

The cDNAs of MdBT2 and MdMYB73 were separately inserted into the $35 \mathrm{~S}::$ pSPYNE-nYFP and 35S::pSPYCEcYFP vectors to form recombinant plasmids MdBT2 $+\mathrm{N}$ and MdMYB73 $+\mathrm{C}$, respectively. Subsequently, these recombinant plasmids were transformed into $A$. tumefaciens LBA4404 (ref. ${ }^{38}$ ). The transformed Agrobacterium suspension was infiltrated into Nicotiana benthamiana leaves. A YFP fluorescent signal was detected to identify interactions using a confocal laser scanning microscope (Zeiss LSM 510 Meta, Jena, Germany) ${ }^{29}$.

\section{Protein degradation assays}

Proteins of three types of apple calli (35S::anti-MdBT2, wild type (WT)), and 35S::MdBT2-MYC) were extracted in degradation buffer ( $25 \mathrm{mM}$ Tris, $5 \mathrm{mM}$ DTT, $10 \mathrm{mM}$ $\mathrm{NaCl}, 10 \mathrm{mM} \mathrm{MgCl}_{2}, 4 \mathrm{mM}$ PMSF, and $10 \mathrm{mM} \mathrm{ATP}$ ) and coincubated with MdMYB73-HIS protein at $22^{\circ} \mathrm{C}$. Subsequently, these samples were collected after $0,2,4$, and $6 \mathrm{~h}$. The reaction of these samples was then stopped by adding $2 \times$ sodium dodecyl sulfate (SDS) loading buffer (0.06 M Tris-HCl, pH 6.8; 1\% SDS; $1 \%$ 2-mercaptoethanol; $10 \%$ glycerol; and $0.025 \%$ bromophenol blue). These samples were then examined by western blotting after being subjected to SDS-polyacrylamide gel electrophoresis (PAGE) on 10\% gels, and transferred to polyvinylidene fluoride (PVDF) membranes (Roche) and an anti-HIS (Beyotime) antibody ${ }^{36}$. For the proteasome inhibitor experiments, three types of apple calli were treated with $50 \mu \mathrm{M}$ MG132 and then extracted. Subsequently, the extracts were coincubated with MdMYB73HIS protein ${ }^{24}$.

\section{Protein ubiquitination assays}

Protein ubiquitination experiments were performed both in vivo and in vitro. For the in vitro ubiquitination assays, a protein ubiquitination solution buffer $(50 \mathrm{mM}$ Tris (pH 7.5), $2 \mathrm{mM}$ DTT, $50 \mathrm{mM} \mathrm{MgCl}_{2}, 2 \mathrm{mM}$ ATP, $100 \mathrm{ng}$ rabbit E1, $100 \mathrm{ng}$ human E2, and $1 \mu \mathrm{g}$ ubi) was prepared as previously described ${ }^{24}$. The MdBT2-MYC active protein was extracted from the 35S::MdBT2-MYC apple calli using a Pierce classic protein A IP Kit (Thermo
Fisher Scientific, San Jose, CA, USA). Mixtures including the buffer and MdMYB73-HIS protein were incubated with or without active MdBT2-MYC protein at $30^{\circ} \mathrm{C}$ for the ubiquitination assays performed with western blotting. These samples were separated by $10 \%$ SDS-PAGE and blotted onto PVDF membranes. The gel blots were probed with anti-HIS and anti-ubi (Sigma Aldrich) antibodies, and were observed via chemiluminescence with an ECL Plus detection kit (Millipore) according to the manufacturer's instructions ${ }^{39}$.

For the in vivo ubiquitination assays, 35S::MdMYB73GFP and 35S::MdMYB73-GFP + 35S::MdBT2-MYC were treated with $50 \mu \mathrm{M}$ MG132, and then extracted using a Pierce Classic Protein A IP Kit. The resulting extractions were examined by western blotting. The proteins were separated by $10 \%$ SDS-PAGE and electroblotted onto PVDF membranes. The gel blots were probed with anti-GFP (Beyotime) and anti-ubi antibodies, and observed via chemiluminescence with the ECL Plus detection kit (Millipore) according to the manufacturer's instructions ${ }^{39}$.

\section{qRT-PCR assays}

Total RNA was extracted using an RNA Plant Plus reagent kit (Tiangen, Beijing, China) according to the manufacturer's instructions. cDNA was synthesized with a PrimeScript ${ }^{\mathrm{TM}} \mathrm{RT}$ reagent kit (TaKaRa, Dalian, China) following the manufacturer's instructions ${ }^{38}$. For qRT-PCR assays, the reactions were carried out with SYBR Green PCR Master Mix in an iCycler iQ5 system (Bio-Rad, Hercules, CA, USA) ${ }^{36}$. The cycle threshold (Ct) $2^{-\Delta \Delta \mathrm{Ct}}$ method was used for detecting the transcript levels of malate-related genes, with MdACTIN used as the reference gene $^{40}$. Each sample was analyzed with three biological replicates. All primers used are shown in Supplementary Table 1.

\section{Transient expression assay in apple fruit}

For antisense suppression, 200-300 bp sequences of the $M d M Y B 73$ and MdBT2 genes in antisense orientation were inserted into the TRV2 vector, and TRV1 was used as an auxiliary vector ${ }^{30}$. The TRV2-antisense constructs and TRV1 were subsequently transformed into A. tumefaciens strain LBA4404. The TRV2- and TRV1-carrying Agrobacterium was grown overnight until an OD value of 0.6 was reached, and then collected by centrifugation at 6000 r.p.m. The cells were resuspended in $10 \mathrm{mM} \mathrm{MgCl}_{2}$, $10 \mathrm{mM} \mathrm{MES}$, and $200 \mu \mathrm{M}$ acetosyringone and then maintained at room temperature for another $2-3 \mathrm{~h}$ in the dark. The two were mixed at a 5:1 ratio just before injection $^{41}$. The fresh-bagged apple fruits of the "Red Delicious" cultivar were collected $\sim 20$ days before harvest from the orchard and used for transient expression assays. A blue dye was first injected into the apple to observe the 
apple injection pathway. Different infection solutions were subsequently injected into the fruit flesh, and then, the samples were extracted based on the approximate pathway of the diffused dye. qRT-PCR assays were used to detect the expression levels of the different vectors to ensure that transient expression was realized.

\section{Measurement of malate concentration, V-ATPase and V- PPase activity levels, and vacuolar pH}

Samples of apple calli or apple fruit were thoroughly ground in liquid nitrogen, and then extracted with $80 \%$ $(\mathrm{v} / \mathrm{v})$ ice-cold methanol ${ }^{24,42}$. The extracted residue was dissolved in deionized water and filtered using a $0.45-\mu \mathrm{m}$ membrane filter ${ }^{24}$. The malate concentration of the filtrate was measured using high-performance liquid chromatography (HPLC $)^{42}$. The activity levels of VATPase and V-PPase were determined using extracted tonoplast membranes ${ }^{19}$. The release of inorganic phosphate was due to the bafilomycin A1-sensitive ATP hydrolytic activity, the ATP-dependent fluorescence quenching of the $\mathrm{pH}$-sensitive fluorescent probe acridine orange was due to V-ATPase $\mathrm{H}^{+}$transport activity, and the production of inorganic phosphate from pyrophosphate was due to V-PPase transport activity ${ }^{2,43,44}$. Vacuolar $\mathrm{pH}$ measurements were performed using extracted protoplasts from apple calli according to previously described methods ${ }^{45}$. The extracted protoplasts were incubated with the $\mathrm{pH}$-sensitive fluorescent dye 2',7'-bis(2-carboxyethyl)-5(6)-carboxyfluorescein acetoxymethyl ester (BCECF-AM; Molecular Probes, Eugene, $\mathrm{OR}$ ) and then measured using a confocal microscope (Zeiss LSM 510 Meta). Vacuolar pH was quantified using the ratio of 488 and $458 \mathrm{~nm}$ excitation wavelengths via Zeiss LSM confocal software ${ }^{12}$.

\section{GUS analysis}

The 1689-bp promoter fragment upstream of the ATG initiation codon of MdALMT9 was inserted into pCAMBIA1300 and transformed into A. tumefaciens strain LBA4404 to obtain transgenic apple calli (proMdALMT9:: GUS). GUS assays were conducted to verify the effect of MdBT2 on the transcriptional activity of MdALMT9. GUS assays were performed using a fluorescence spectrometer (Thermo Scientific) according to previously described methods ${ }^{34}$.

\section{Statistical analysis}

At least three biological replicates were performed for each experiment. The data are presented as the means \pm standard deviation unless otherwise indicated. Statistical analysis was carried out via Tukey's single factor test using DPS software. For all bar charts, the bars with different letters indicate significantly differences at $P<0.05$.

\section{Results}

The conserved BTB-BACK and R2R3 domains are necessary for the interaction between MdBT2 and MdMYB73 proteins

To determine whether MdBT2 interacts with the MdMYB73 protein, we utilized Y2H assays. The MdBT2 protein contains three conserved domains, including the BTB, BACK, and ZnF_TAZ domains (Fig. 1a and Supplementary Table 2). Therefore, the full-length cDNA of the $M d B T 2$ gene was divided into five fragments: MdBT2 $2^{\text {BTB-BACK }}, \mathrm{MdBT}^{\mathrm{BTB}}, \mathrm{MdBT}^{\mathrm{BACK}}$, MdBT2 $2^{\text {BACK-ZnF_TAZ, and } M d B T 2} 2^{\text {ZnF_TAZ }_{-}}$(Fig. 1a). Subsequently, the full-length cDNA and five truncated mutants of the $M d B T 2$ gene were independently inserted into the pGBT9 vector as the bait vectors, while the fulllength cDNA of $M d M Y B 73$ was inserted into the pGAD424 vector as the prey vector. The six combinations of bait and prey vectors were transformed into yeast for $\mathrm{Y} 2 \mathrm{H}$ assays. The results indicated that both the full-length MdBT2 and truncated mutant $\mathrm{MdBT} 2^{\text {BTB-BACK }}$ interacted with the full-length MdMYB73 protein (Fig. 1a).

To further examine the specific interaction region of MdMYB73 with the MdBT2 protein, the $M d M Y B 73$ gene was also divided into four fragments: MdMYB73 ${ }^{\mathrm{R} 2 \mathrm{R} 3}$ (amino acids 1-131), MdMYB73 ${ }^{\mathrm{R} 2}$ (amino acids 1-80), MdMYB73 $^{\text {R3 }}$ (amino acids 81-131), and MdMYB73 ${ }^{\text {None }}$ (amino acids 132-242). The full-length cDNA and four truncated mutants of the $M d M Y B 73$ gene were independently inserted into the pGAD424 vector as the prey vectors. These different combinations of prey vectors with the full-length MdBT2 prey vector were transformed into yeast for use with $\mathrm{Y} 2 \mathrm{H}$ assays. The results showed that both full-length MdMYB73 and truncated mutant MdMYB73 ${ }^{\mathrm{R} 2 \mathrm{R} 3}$ interacted with the full-length MdBT2 protein (Fig. 1b).

To further assess the interaction between MdBT2 and MdMYB73, recombinant MdBT2-GST protein and recombinant MdMYB73-HIS protein were subjected to in vitro GST pull-down assays, while the GST protein and recombinant MdMYB73-HIS protein were used as negative controls. The results showed that the GST-tagged MdBT2 protein interacted with the HIS-tagged MdMYB73 protein (Fig. 1c). In addition, bimolecular fluorescence complementation (BiFC) assays were conducted using $N$. benthamiana leaves to verify the interaction between MdBT2 and MdMYB73. MdBT2 and MdMYB73 were fused to the $\mathrm{N}$-terminal and $\mathrm{C}$-terminal fragments of YFP to construct the recombinant $\mathrm{MdBT} 2+\mathrm{N}$ and MdMYB73 $+\mathrm{C}$ vectors, respectively. As a result, a nuclear-localized signal was detected in tobacco leaf cells coexpressing the MdBT2 $+\mathrm{N}$ and MdMYB73 + $C$ vectors, whereas the other combinations did not show this signal (Fig. 1d). 
(a)

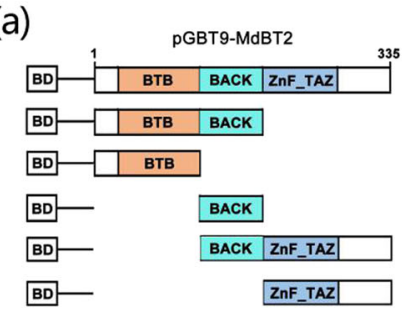

(c)

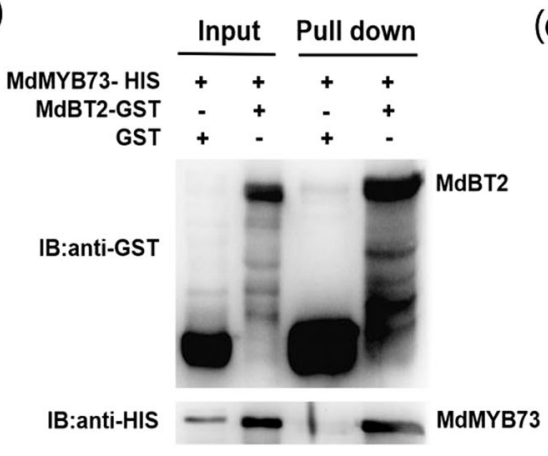

(b)

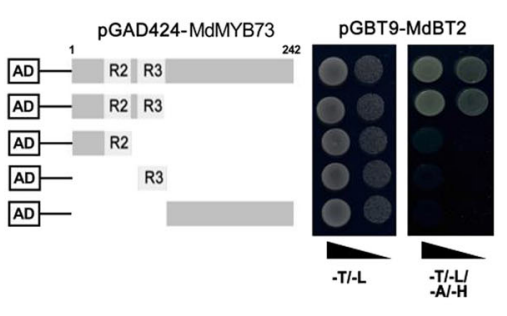

(d)

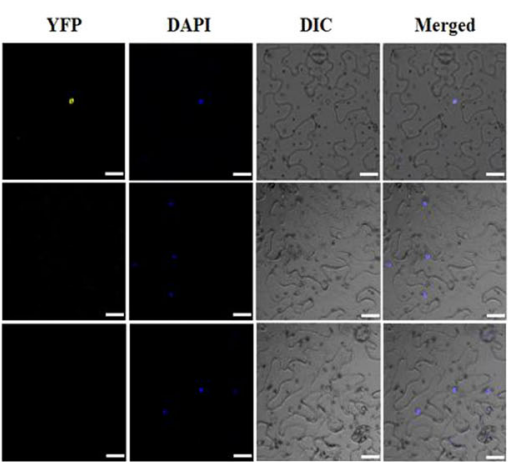

Fig. 1 MdBT2 interacts with MdMYB73. a, b The interaction between MdBT2 and MdMYB73 in a Y2H assay. The full-length cDNA and five truncated mutants of the MdBT2 gene (a) were inserted into the pGBT9 vector. The full-length cDNA of MdMYB73 (a) was inserted into the pGAD424 vector. Panels show the different interactions, as indicated by yeast growth (a). The full-length cDNA and four truncated mutants of the MdMYB73 gene (b) were inserted into the pGAD424 vector. The full-length cDNA of the MdBT2 gene (b) was inserted into the pGBT9 vector. Panels show the interactions as indicated by yeast growth (b). c MdBT2 interacted with MdMYB73, as indicated by pull-down assay. MdBT2-GST expression in E. coli resulted in the precipitation of MdMYB73-HIS, with the anti-GST antibody and anti-HIS antibody. GST alone was used as the control. $\mathbf{d}$ MdBT2 interacted with MdMYB73 in a BiFC assay using tobacco leaf cells. MdBT2 + N and MdMYB73 + C interacted in the nucleus of tobacco leaf cells. Scale bar $=20 \mu \mathrm{m}$. The data were obtained from at least two biological replicates

Taken together, these results suggested that MdBT2 physically interacts with the MdMYB73 protein, and the interaction between these two proteins requires conserved BTB-BACK and R2R3 domains.

\section{MdBT2 affects MdMYB73 protein stability}

Given that the ubiquitination-related MdBT2 scaffold protein influences the stability of its targets ${ }^{24,29,36,39}$, cellfree degradation assays were performed to examine the stability of the MdMYB73 protein. Three types of apple calli, WT, MdBT2-overexpressing apple calli (35S:: MdBT2), and MdBT2-suppressing apple calli (35S::antiMdBT2), were incubated with purified MdMYB73-HIS protein. As shown in Fig. 2a, b and Supplementary Fig. 1, the MdMYB73-HIS protein was degraded faster in the 35S::MdBT2 apple calli than in the WT control, while the degradation was slower in the 35S::anti-MdBT2 apple calli compared to that of the WT control.

Subsequently, the proteasome inhibitor MG132 was added to determine the MdBT2-mediated stability of the MdMYB73 protein. The cell-free degradation assays showed that MdMYB73-HIS protein abundance was unchanged in the three types of apple calli in the presence of MG132 (Fig. 2c, d and Supplementary Fig. 2).

Overall, these results suggested that MdBT2 influenced the stability of the MdMYB73 protein through the $26 \mathrm{~S}$ proteasome pathway.

\section{MdBT2 facilitates the degradation of MdMYB73 protein via the ubiquitin/26S proteasome pathway}

To confirm that MdBT2 degraded the MdMYB73 protein through the ubiquitin/26S proteasome pathway, in vitro immunoprecipitation assays were conducted using anti-HIS and anti-ubi antibodies. MdBT2-MYC active proteins extracted from 35S::MdBT2-MYC were coincubated with MdMYB73-HIS, E1, E2, and ubi in vitro for $24 \mathrm{~h}$. Immunoblotting assays showed that higher amounts of high-molecular mass forms of MdMYB73 protein were detected in immunoprecipitated mixtures, using anti-HIS and anti-ubi antibodies compared with the amounts in the samples without MdBT2-MYC protein added (Fig. 3a, b).

Furthermore, in vivo ubiquitination assays were performed using two types of transgenic apple calli 

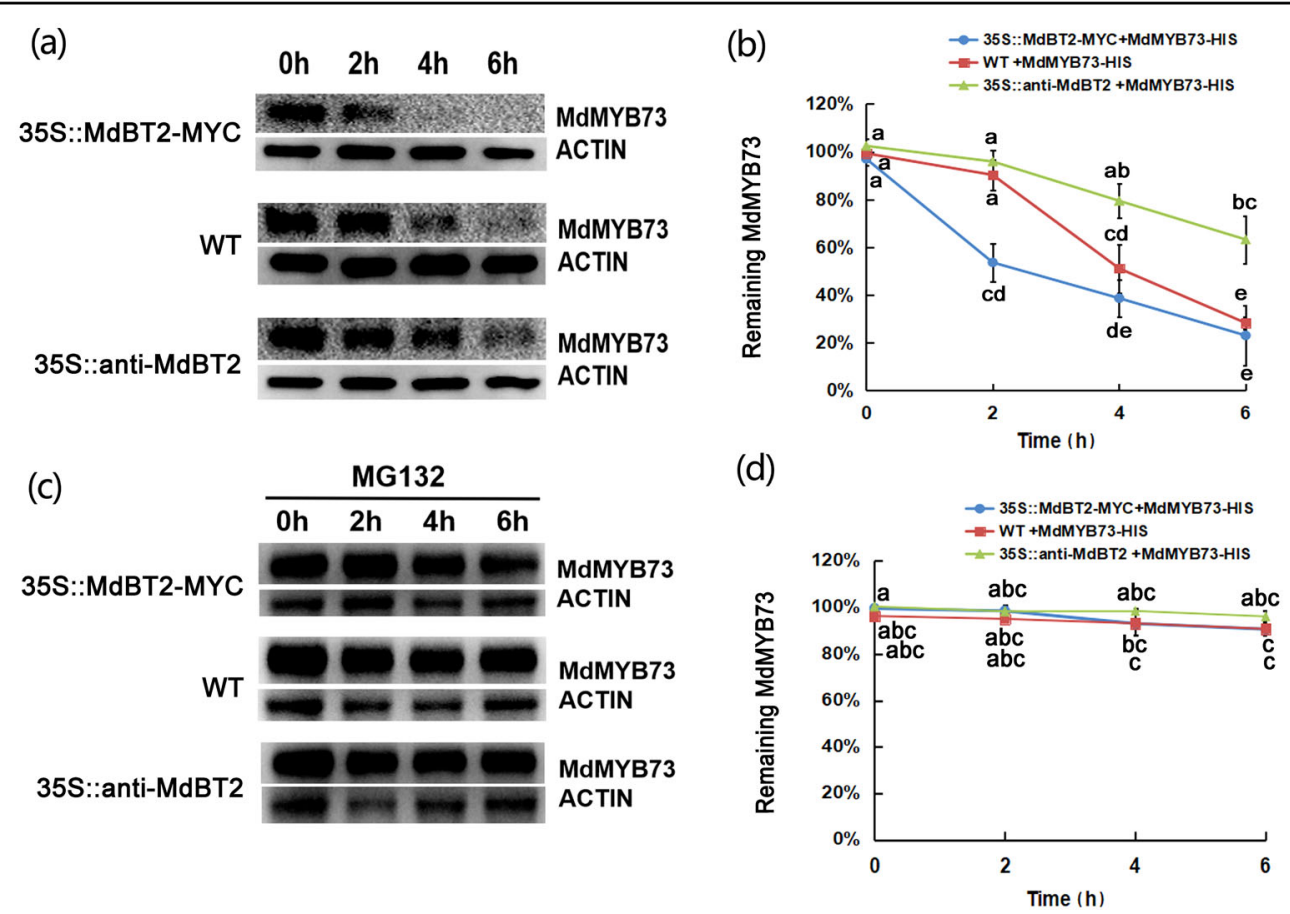

Fig. 2 MdBT2 affects the stability of the MdMYB73 protein. a MdBT2 degraded MdMYB73 in vitro. Cell-free degradation assays were performed to examine the stability of the MdMYB73 protein. Three types of apple calli (WT, 35S::MdBT2, and 35S::.anti-MdBT2) were coincubated with MdMYB73HIS protein at $22^{\circ} \mathrm{C}$ for the times indicated. b Degradation curve analysis of MdMYB73-HIS. c The stability of the MdMYB73 protein after MG132 treatment. WT, 355::MdBT2, and 355::anti-MdBT2 transgenic apple calli were treated with 50 mM MG132, and then incubated with MdMYB73-HIS at $22^{\circ} \mathrm{C}$ for the times indicated. $\mathbf{d}$ Degradation curve analysis of MdMYB73-HIS upon MG132 treatment. The experiments were performed for three biological replicates, and the data are presented as the means \pm standard deviation. Bars with different letters indicate significant differences at $P<0.05$

(35S::MdMYB73-GFP and 35S::MdMYB73-GFP + 35S:: MdBT2-MYC). The immunoprecipitated protein was subsequently detected using anti-GFP and anti-ubi antibodies (Fig. 3c, d). The abundance of polyubiquitinated MdMYB73-GFP protein in 35S::MdMYB73-GFP + 35S:: MdBT2-MYC apple calli was much higher than it was in the single 35S::MdMYB73-GFP apple calli. These results suggested that MdBT2 promoted the degradation of MdMYB73 protein via the ubiquitin/26S proteasome pathway.

\section{MdMYB73 is required for $M d B T 2$ to control malate accumulation and vacuolar $\mathrm{pH}$ in apples}

To characterize whether and how MdBT2 controls malate accumulation, a viral vector-based method was applied by injection into apple fruits. A blue dye was initially injected into the apple fruits to observe the injection pathway (Supplementary Fig. 3). Subsequently, four types of viral constructs (TRV, TRV-MdBT2, TRVMdMYB73, and TRV-MdBT2 + TRV-MdMYB73) were obtained for fruit infiltration assays (Fig. 4a). The qRT-PCR assays showed that the relative transcript levels of the MdBT2 gene were 0.58 and 0.75 after being separately infiltrated with TRV-MdBT2 and TRV-MdBT2 + TRV-
MdMYB73, respectively, compared with TRV. The relative transcript levels of the $M d M Y B 73$ gene were 0.57 and 0.78 after being separately infiltrated with TRV-MdMYB73 and TRV-MdBT2 + TRV-MdMYB73, respectively, compared with TRV (Fig. 4b). These results indicated that the infiltration assays were successful. As the direct downstream target genes of the MdMYB73 TF, the transcript levels of $M d V H A-A, M d V H P 1$, and MdALMT9 decreased with the decrease in $M d M Y B 73$ gene expression. The transcript levels of $M d M Y B 73$ and its direct downstream genes were negatively associated with the $M d B T 2$ gene transcript level (Fig. 4b). Subsequently, the hydrolytic and proton-pumping activity levels of V-ATPase and V-PPase were determined. V-ATPase and V-PPase activity levels in the TRVMdMYB73-injected areas were much lower than those in the TRV control. However, TRV-MdBT2-injected areas had much higher V-ATPase and V-PPase activities than those of the TRV control (Fig. 4d-f). Furthermore, TRVMdMYB73 counteracted the effect of TRV-MdBT2 in TRV-MdBT2 + TRV-MdMYB73-injected areas (Fig. 4d-f), suggesting that MdBT2 influenced V-ATPase and V-PPase activities, depending on the presence of MdMYB73. As expected, the malate concentration was in agreement with the V-ATPase and V-PPase activity levels; the malate 


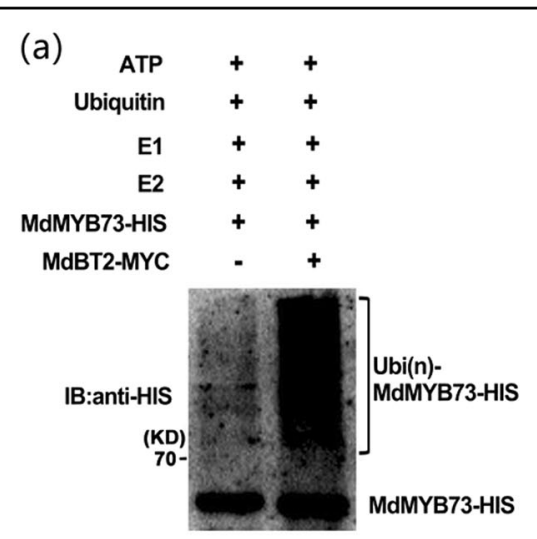

(c)

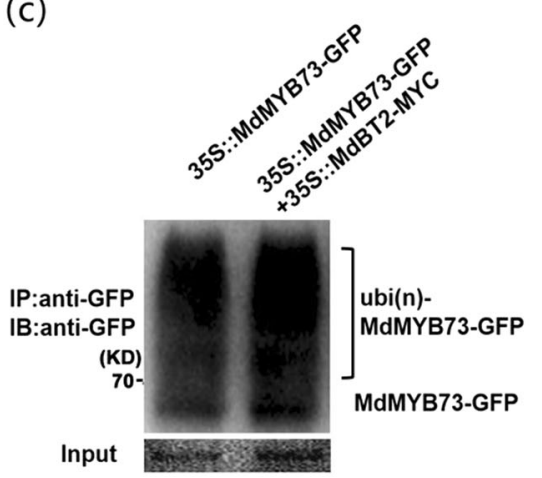

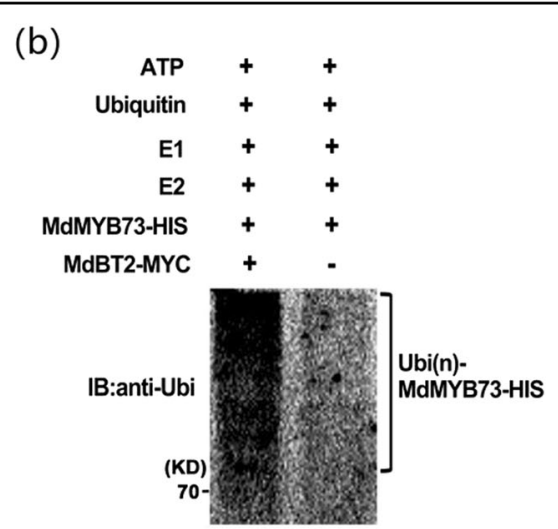

(d)

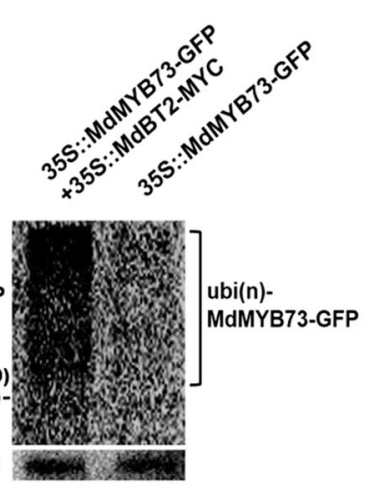

Fig. 3 MdBT2 facilitates ubiquitination and degradation of the MdMYB73 protein. $\mathbf{a}, \mathbf{b}$ Ubiquitination assays were performed in vitro. The MdBT2-MYC active protein extracted from 355::MdBT2-MYC was incubated with MdMYB73-HIS, E1, E2, and ubi at $30^{\circ} \mathrm{C}$ for immunoprecipitation, using anti-HIS (a) and anti-ubi (b) antibodies. In a, the left side lane had two nonspecific bands. c, d Ubiquitination assays in vivo were measured using 355 :.: MdMYB73-GFP and 355::MdMYB73-GFP + 35S::MdBT2-MYC. Anti-GFP (c) and anti-ubi (d) antibodies were used to examine immunoprecipitation. The assays were performed with at least two replicates. IP Immunoprecipitated, IB immunoblotted

content was reduced in the TRV-MdMYB73 and TRVMdBT2 + TRV-MdMYB73-injected areas compared to that of the control (ab), and only MdMYB73-TRV (c) showed reduced malate concentration. MdBT2-TRV (a) and MdBT2-TRV + MdMYB73-TRV (bc) were not significantly different from the control (Fig. 4c).

Four types of apple calli (WT, 35S::anti-MdBT2, 35S:: anti-MdMYB73, and 35S::anti-MdBT2 + 35S::antiMdMYB73) were used to study the regulation of malate accumulation. The $M d B T 2$ and $M d M Y B 73$ genes were successfully suppressed in the corresponding calli compared to the WT control (Fig. 5a), indicating that the genetic transformation in apple calli was successful. As downstream genes of MdMYB73, the MdVHA-A, $M d V H P 1$, and MdALMT9 genes exhibited reduced transcript levels in the 35S::anti-MdMYB73 and 35S::antiMdBT2 + 35S::anti-MdMYB73 samples, and they were increased in the 35S::anti-MdBT2 sample (Fig. 5a).

Next, malate concentration and the hydrolytic and proton-pumping activity levels of V-ATPase and V-PPase were also examined. This analysis revealed that malate concentration V-ATPase and V-PPase activity levels in the 35S::anti-MdMYB73 apple calli were much lower than those in the WT control. However, 35S::anti-MdBT2 calli had a much higher malate concentration and V-ATPase and V-PPase activity levels compared with those of the WT control (Fig. 5b-e). Moreover, 35S::anti-MdMYB73 counteracted the effect of 35S::anti-MdBT2 in the 35S:: anti-MdBT2 + 35S::anti-MdMYB73 calli (Fig. 5b-e), suggesting that MdBT2 influenced malate concentration, as well as V-ATPase and V-PPase activity levels, depending on the presence of MdMYB73. In addition, the vacuolar $\mathrm{pH}$ in the apple calli was measured using a BCECF-AM ratiometric fluorescent $\mathrm{pH}$ indicator. As shown in Fig. 5f, g, the average vacuolar $\mathrm{pH}$ in the WT, 35S::anti-MdMYB73, and 35S::anti-MdBT2 calli was 4.28, 4.70 , and 4.03, respectively, while the vacuolar $\mathrm{pH}$ in the 35S::anti-MdBT2 + 35S::anti-MdMYB73 calli was 4.41 . These results indicated that only the silencing of $M d M Y B 73$ increased vacuolar $\mathrm{pH}$, while the silencing of $M d B T 2$ or $M d B T 2$, and $M d M Y B 73$ genes did not change vacuolar $\mathrm{pH}$ compared to that of the control. 


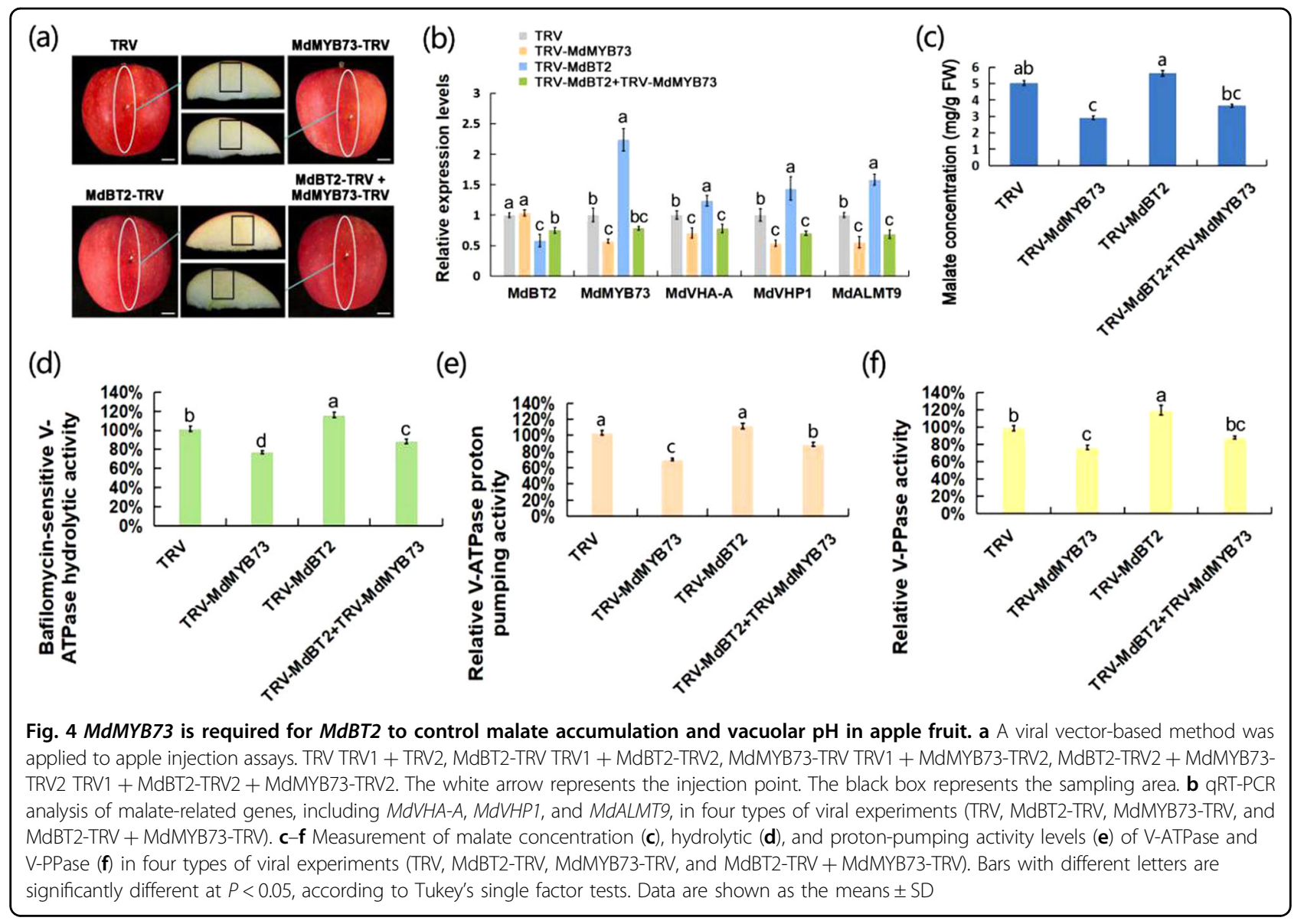

These results support our hypothesis that MdMYB73 is required for MdBT2 to control malate accumulation and vacuolar $\mathrm{pH}$.

\section{MdBT2 negatively regulates the MdMYB73-downstream gene MdALMT9}

GUS assays were performed to verify the effect of MdBT2 on the transcriptional activity of MdALMT9 using proMdALMT9::GUS, with proMdALMT9::GUS + 35S::MdMYB73 used to detect GUS enzyme activity. This analysis revealed that proMdALMT9::GUS + 35S:: MdMYB73 generated higher GUS enzyme activity than proMdALMT9::GUS alone (Fig. 6a, b). However, GUS enzyme activity decreased when 35S::MdBT2 was transformed into proMdALMT9::GUS + 35S::MdMYB73, suggesting that MdBT2 negatively regulated MdALMT9 transcriptional activity via the MdBT2-MdMYB73MdALMT9 pathway.

\section{Discussion}

Malate is a pivotal metabolite that is involved in regulating osmotic pressure, $\mathrm{pH}$ homeostasis, and stress resistance, and it significantly influences fruit quality ${ }^{2,7}$. A previous study has shown that the BTB-BACK-TAZ protein MdBT2 ubiquitinates the bHLH TF MdCIbHLH1 to regulate malate accumulation and vacuolar acidification $^{24}$. In this study, we discovered that malate accumulation and vacuolar acidification are also regulated, by MdBT2 ubiquitinating the MYB TF MdMYB73 in apple.

Acting as an important posttranslational modification, ubiquitination plays an important role in regulating protein activity in eukaryotes, with significant impacts on plant growth and development ${ }^{34,46-48}$. There are some studies on the transcriptional regulation of malate accumulation, but posttranslational regulatory mechanisms, such as ubiquitination have not been studied extensively $y^{2,5,12,24}$. In addition to the previously identified MdCIbHLH1 TF, we found that an MdMYB73 TF was ubiquitinated by MdBT2 to regulate malate accumulation and vacuolar acidification. MdCIbHLH1 and MdMYB73 both function as TFs and are targets of MdBT2. Remarkably, MdCIbHLH1 also interacted with MdMYB73 to form the MBW complex, which enhanced the effect on downstream target genes to modulate malate accumulation and vacuolar $\mathrm{pH}^{2}$.

As a scaffold protein, BT2 recruits E3 ubiquitin ligases, such as CUL3 and RBX1 to form a complex for the ubiquitination and degradation of target proteins ${ }^{26,27}$. 


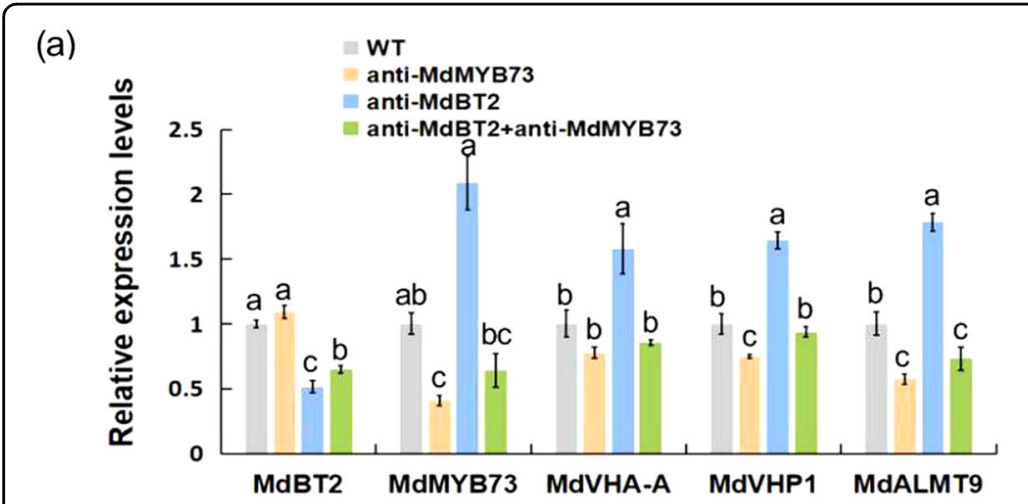

(c)

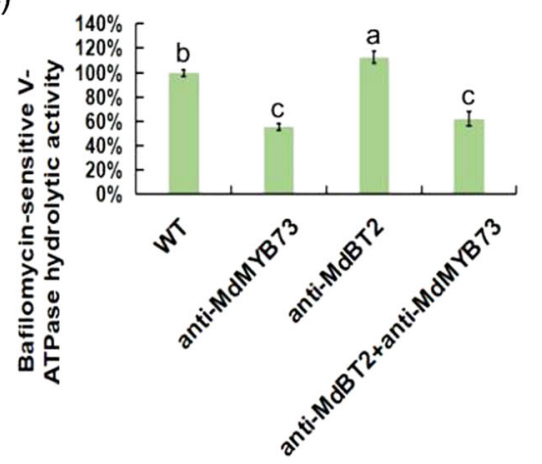

(d)

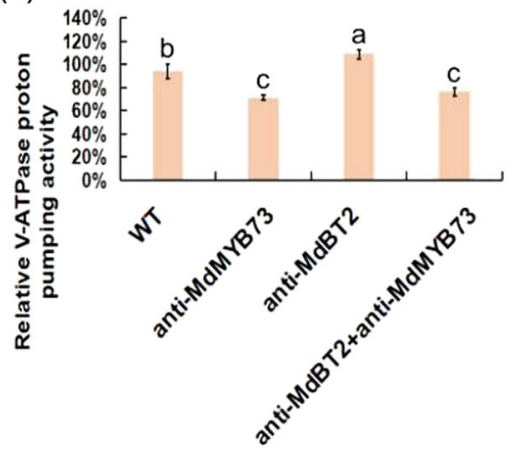

(b)

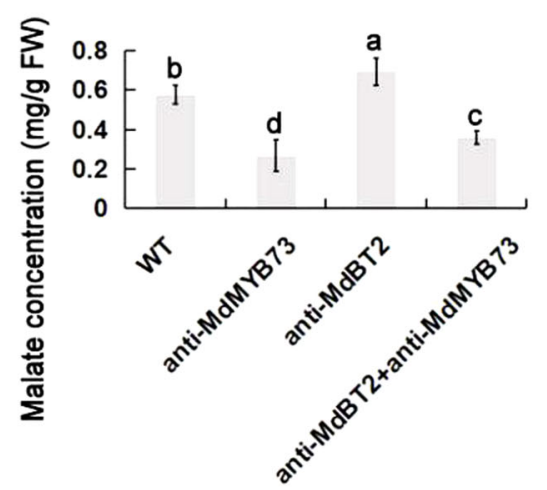

(e)

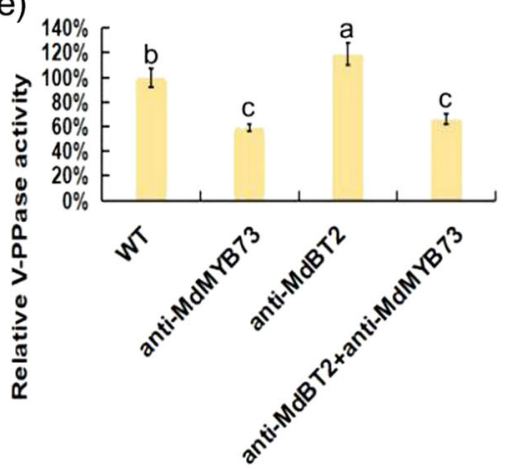

(f)

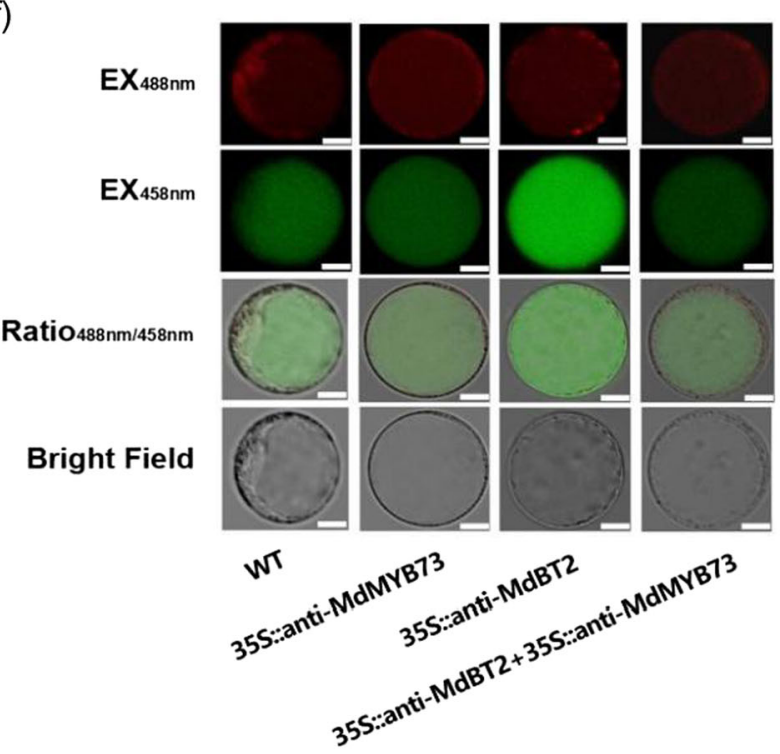

(g)

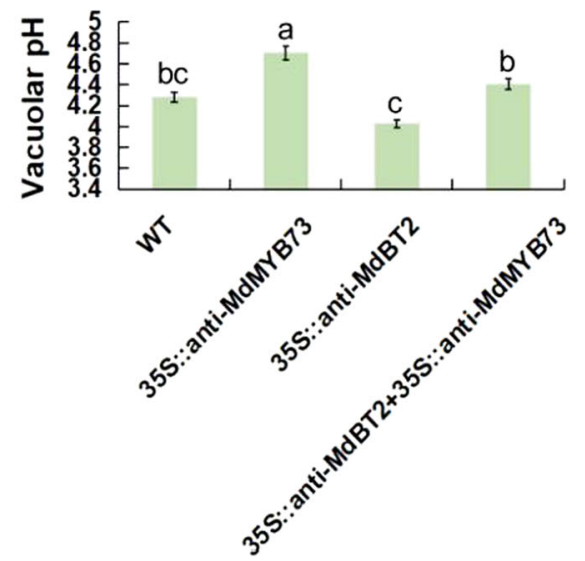

Fig. 5 MdMYB73 is required for MdBT2 to control malate accumulation and vacuolar pH in apple calli. a Expression analysis of malate-related genes, MdVHA-A, MdVHP1, and MdALMT9, using qRT-PCR in WT and transgenic apple calli (35S::anti-MdMYB73, 35S::anti-MdBT2, and 35S::antiMdBT2 + 35S:::anti-MdMYB73). b The malate concentration in the WT and transgenic apple calli (35S::anti-MdMYB73, 35S::anti-MdBT2, and 35S::antiMdBT2 + 35S::anti-MdMYB73). c-e Hydrolytic (c) and proton-pumping activity levels of V-ATPase (d) and V-PPase (e) in WT and transgenic apple calli (35S::anti-MdMYB73, 35S::anti-MdBT2, and 35S::anti-MdBT2 + 35S::anti-MdMYB73). f, g Emission intensities (f) and quantitative analysis (g) of protoplasts to determine the vacuolar pH in apple calli with BCECF dyes at $488 \mathrm{~nm}$ and $458 \mathrm{~nm}$ (WT, 35S::anti-MdBT2, 35S::anti-MdMYB73, and 35S::anti-MdBT2 + 35S::anti-MdMYB73). Scale bar $=10 \mu \mathrm{m}$. Bars with different letters are significantly different at $P<0.05$, according to Tukey's single factor tests. Data are shown as the means \pm SD 
(a)

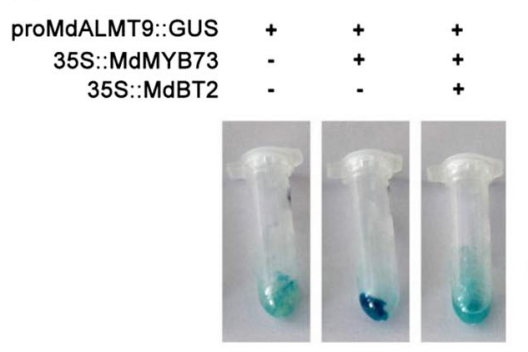

(b)

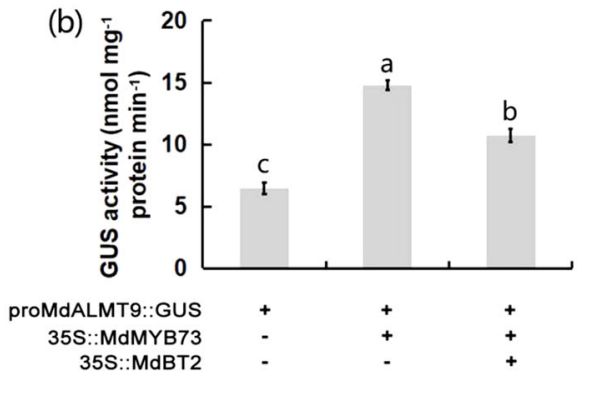

Fig. 6 MdBT2 negatively regulates the MdMYB73-downstream gene MdALMT9. a GUS assays in different apple calli (35S::MdBT2 + 35 S::: MdMYB73 + proMdALMT9::GUS, proMdALMT9::GUS + 355::MdMYB73, and proMdALMT9::GUS). b GUS activity measured using a fluorescence spectrometer with excitation at $365 \mathrm{~nm}$ and emission at $455 \mathrm{~nm}$ in different apple calli (35S::MdBT2 + 35 S::MdMYB73 + proMdALMT9::GUS, proMdALMT9::GUS + 35S::MdMYB73, and proMdALMT9::GUS). Bars with different letters are significantly different at $P<0.05$, according to Tukey's single factor tests. Data are shown as the means \pm SD

Recently, MdBT2 was found to be involved in ubiquitination to regulate iron homeostasis depending on the ubiquitin ligase MdCUL3 (ref. ${ }^{39}$ ). MdBT2 facilitated the ubiquitination of MdMYB1 to regulate the anthocyanin production through an MdCUL3-independent pathway ${ }^{36}$. Interestingly, the TAZ domain of MdBT2 was required for the interaction between MdBT2 and MdbHLH104, which regulated iron homeostasis, while the BACK domain of MdBT2 was required for the MdBT2MdMYB1 protein interaction ${ }^{36,39}$. In the current study, we found that the conserved BTB-BACK domain was necessary for the interaction between MdBT2 and MdMYB73 proteins (Fig. 1). Therefore, we speculate that MdBT2 can accelerate the ubiquitination of MdMYB73, possibly through an MdCUL3-independent pathway. However, the determination of whether MdBT2 ubiquitinates MdMYB73 by recruiting a ubiquitin E3 ligase (RBX1 or others) remains for further examination.

MdMYB73 promotes malate accumulation by activating proton pumps and secondary transporters, such as MdALMT9, MdVHA-A, and MdVHP1 (ref. ${ }^{2}$ ). In addition, another MYB TF (MdMYB1) plays a central role in malate accumulation by targeting MdVHA-Bs, MdVHAE, MdVHP1, and MdtDT ${ }^{12}$. Although MdMYB73 and MdMYB1 have similar functions in regulating malate accumulation, the downstream target genes of MdMYB1 and MdMYB73 differ. These findings clearly imply that MYB TFs play important roles in malate accumulation, but by different pathways. Interestingly, MdBT2 interacts with both MdMYB1 and MdMYB73 (Fig. 1) ${ }^{36}$. MdMYB1 regulates both anthocyanin and malate accumulation, and MdBT2 regulates the accumulation of MdMYB1mediated anthocyanin ${ }^{12,36}$. However, whether MdBT2 affects malate accumulation through MdMYB1 still needs further study. The role of MdALMT9 in regulating malate accumulation has been clearly demonstrated ${ }^{2,5,7,20}$, and MdMYB73 regulates malate accumulation by activating the transcription of MdALMT9 (ref. ${ }^{2}$ ). In this study, the effect of MdBT2 on malate accumulation through MdMYB73-MdALMT9 was confirmed by the lower GUS enzyme activity seen in 35S::MdBT2 + 35S::MdMYB73 + proMdALMT9::GUS (Fig. 6). Compared to the anthocyanin accumulation regulated by MdBT2-MdMYB1 $\left(\right.$ ref. $\left.{ }^{36}\right)$, malate accumulation was regulated by MdBT2MdMYB73. It is currently unknown whether MdMYB73 affects anthocyanin accumulation or whether MdBT2 also regulates MdMYB73-mediated anthocyanin accumulation; therefore, further studies are needed.

The BTB-BACK-TAZ protein, MdBT2, plays various roles in the integration of signals involving light, temperature, carbon and nitrogen nutrition, hormones, and stresses in plants ${ }^{28,29,36,39}$. In apple, the ABA-responsive protein MdBT2 interacts directly with MdbHLH93 and degrades the MdbHLH93 protein by the ubiquitin/26S proteasome pathway, resulting in delayed leaf senescence $^{49}$. In addition, MdBT2 is also a nitrate-responsive protein that regulates anthocyanin biosynthesis by interacting with the MdMYB1 TF in apple ${ }^{36}$. MdBT2 also regulates plant cold tolerance by promoting the degradation of the MdMYB23 protein ${ }^{29}$. Previous research indicating that MdBT2 affects malate accumulation by targeting MdMYB73 was also verified in this study. Thus, we propose that different external environmental factors, such as nitrate signaling, light or cold, can regulate malate accumulation.

Taken together, our discoveries and those of previous studies can be integrated into a working model (Fig. 7). This working model depicts a mechanism, in which the BTB-BACK-TAZ protein MdBT2 regulates malate accumulation and vacuolar $\mathrm{pH}$. With environmental stimuli (such as nitrate signaling), MdBT2 ubiquitinates MdCIbHLH1 and MdMYB73 to target them for degradation, which reduces the transcription of malateassociated genes by MdMYB73, leading to lower malate 


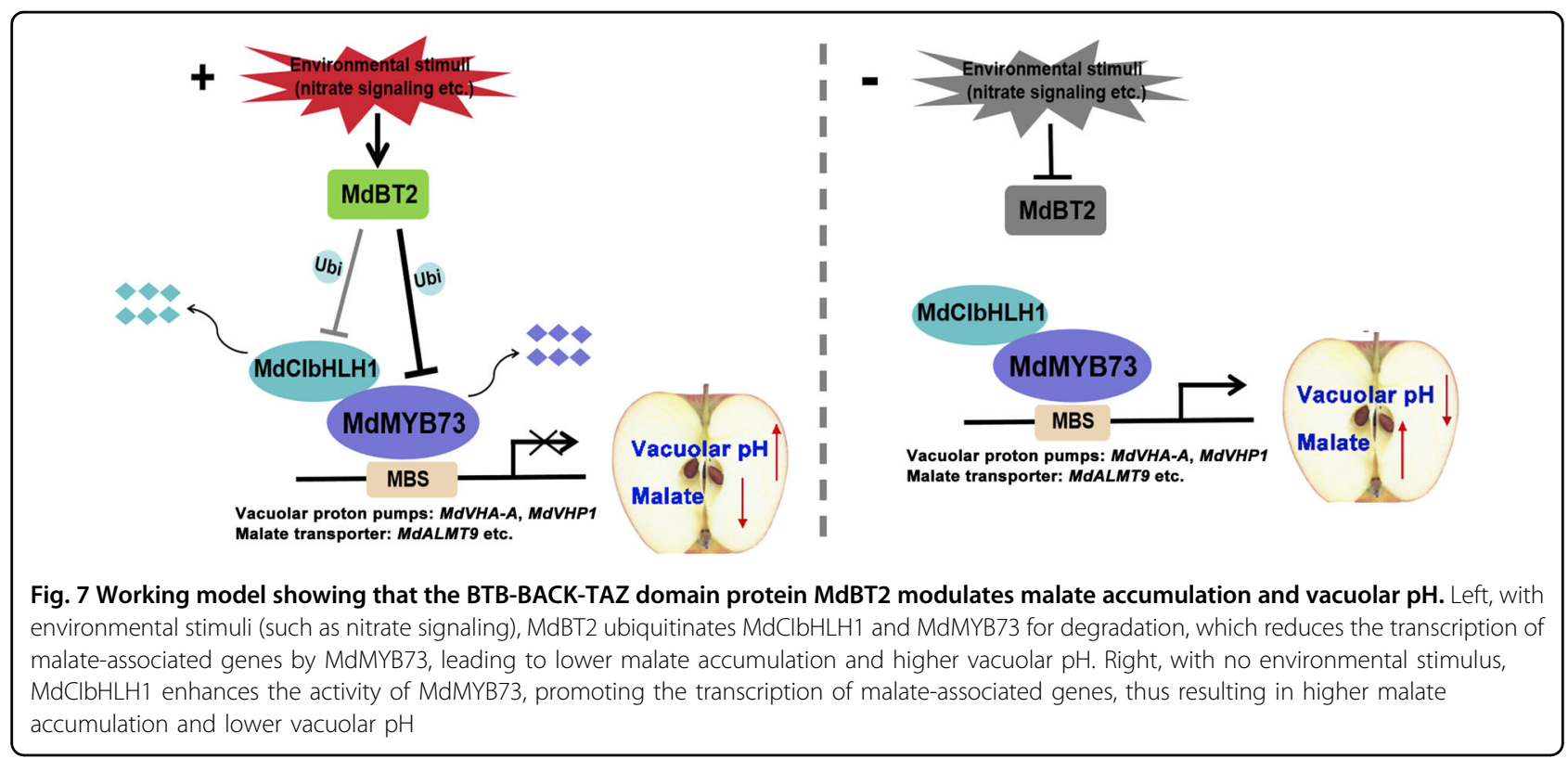

accumulation and high vacuolar $\mathrm{pH}$. When there are no environmental stimulus, MdCIbHLH1 enhances the activity of MdMYB73, promoting the transcription of malate-associated genes, thus resulting in high malate accumulation and lower vacuolar $\mathrm{pH}$. Overall, our findings provide new insights into the molecular mechanism and regulatory pathway of the ubiquitination of relevant TFs, affecting malate accumulation and expand the current understanding of malate regulation mechanisms. This information may be of particular significance in guiding traditional breeding programs and fruit tree molecular breeding, as well as improving fruit quality and stress tolerance.

\section{Acknowledgements}

We would like to thank Prof. Takaya Moriguchi of the National Institute of Fruit Tree Science, Japan, for the "Orin" apple calli. We would like to thank TopEdit (www.topeditsci.com) for its linguistic assistance during the preparation of this manuscript. This work was supported by grants from the National Key Research and Development Program of China (2018YFD1000200), the National Natural Science Foundation of China (31972375), Ministry of Agriculture (CARS27), and Shandong Province (SDAIT-06-03).

\section{Author contributions}

Y.-J.H. and D.-G.H. planned and designed the research. Q.-Y.Z., D.-G.H., K.-D.G., J.-H.W., J.-Q.Y., X.-F.W., S.Z., and C.-X.Y. performed experiments, conducted fieldwork, and analyzed the data. Q.-Y.Z., D.-G.H., and Y.-J.H. wrote the manuscript.

\section{Conflict of interest}

The authors declare that they have no conflict of interest.

Supplementary Information accompanies this paper at (https://doi.org/ 10.1038/s41438-020-00384-z)

Received: 20 March 2020 Revised: 14 July 2020 Accepted: 27 July 2020 Published online: 02 September 2020

\section{References}

1. Finkemeier, I. \& Sweetlove, L. J. The role of malate in plant homeostasis. F1000 Biol. Rep. 1, 47 (2009).

2. $\mathrm{Hu}, \mathrm{D}$. G. et al. The R2R3-MYB transcription factor MdMYB73 is involved in malate accumulation and vacuolar acidification in apple. Plant J. 91, 443-454 (2017).

3. Yao, Y. X., Li, M., Liu, Z., Hao, Y. J. \& Zhai, H. A novel gene, screened by cDNAAFLP approach, contributes to lowering the acidity of fruit in apple. Plant Physiol. Biochem. 45, 139-145 (2007).

4. Bai, Y., Dougherty, L., Cheng, L. L. \& Xu, K. N. A co-expression gene network associated with developmental regulation of apple fruit acidity. Mol. Genet. Genomics. 290, 1247-1263 (2015).

5. Ye, J. et al. An InDel in the promoter of Al-ACTIVATED MALATE TRANSPORTER9 selected during tomato domestication determines fruit malate contents and aluminum tolerance. Plant Cell 29, 2249-2268 (2017).

6. Butelli, E. et al. Noemi controls production of flavonoid pigments and fruit acidity and illustrates the domestication routes of modern citrus varieties. Curr. Biol. 29, 158-164 (2019).

7. Li, C. L. et al. Apple ALMT9 requires a conserved C-terminal domain for malate transport underlying fruit acidity. Plant Physiol. 182, 992-1006 (2020).

8. Etienne, A., Génard, M., Lobit, P., Mbeguié-A-Mbéguié, D. \& Bugaud, C. What controls fleshy fruit acidity? A review of malate and citrate accumulation in fruit cells. J. Exp. Bot. 64, 1451-1469 (2013).

9. Sweetman, C., Deluc, L. G., Cramer, G. R., Ford, C. M. \& Soole, K. L. Regulation of malate metabolism in grape berry and other developing fruits. Phytochemistry 70, 1329-1344 (2009).

10. Sweetlove, L. J., Beard, K. F., Nunes-Nesi, A., Fernie, A. R. \& Ratcliffe, R. G. Not just a circle: flux modes in the plant TCA cycle. Trends Plant Sci. 15, $462-470$ (2010).

11. Centeno, D. C. et al. Malate plays a crucial role in starch metabolism, ripening, and soluble solid content of tomato fruit and affects postharvest softening. Plant Cell 23, 162-184 (2011).

12. Hu, D. G. et al. MdMYB1 regulates anthocyanin and malate accumulation by directly facilitating their transport into vacuoles in apples. Plant Physiol. 170, 1315-1330 (2016).

13. Martinoia, E., Maeshima, M. \& Neuhaus, H. E. Vacuolar transporters and their essential role in plant metabolism. J. Exp. Bot. 58, 83-102 (2007).

14. Sze, H., Schumacher, K, Muller, M. L., Padmanaban, S. \& Taiz, L. A simple nomenclature for a complex proton pump: VHA genes encode the vacuolar H-ATPase. Trends Plant Sci. 7, 157-161 (2002).

15. Padmanaban, S., Lin, X., Perera, I., Kawamura, Y. \& Sze, H. Differential expression of vacuolar $\mathrm{H}+$-ATPase subunit $\mathrm{C}$ genes in tissues active in membrane trafficking and their roles in plant growth as revealed by RNAi. Plant Physiol. 134, 1514-1526 (2004) 
16. Rea, P. A. et al. Vacuolar H+-translocating pyrophosphatases: a new category of ion translocase. Trends Biochem. Sci. 17, 348-353 (1992).

17. Maeshima, M. Vacuolar H-pyrophosphatase. Biochim. Biophys. Acta 1465 37-51 (2000)

18. Li, M. et al. Proteomic analysis reveals dynamic regulation of fruit development and sugar and acid accumulation in apple. J. Exp. Bot. 67, 5145-5157 (2016).

19. Terrier, N., Sauvage, F. X. \& Romieu, C. Changes in acidity and in proton transport at the tonoplast of grape berries during development. Planta $\mathbf{2 1 3}$ 20-28 (2001).

20. Bai, Y. et al. A natural mutation-led truncation in one of the two aluminumactivated malate transporter-like genes at the Ma locus is associated with low fruit acidity in apple. Mol. Genet. Genomics 287, 663-678 (2012).

21. Ma, B. et al. A Ma10 gene encoding P-type ATP ase is involved in fruit organic acid accumulation in apple. Plant Biotechnol. J. 17, 674-686 (2019).

22. Jia, D. et al. Apple fruit acidity is genetically diversified by natural variations in three hierarchical epistatic genes: MdSAUR37, MdPP2CH and MdALMTII. Plant J. 95, 427-443 (2018).

23. Duan, N. et al. Genome re-sequencing reveals the history of apple and supports a two-stage model for fruit enlargement. Nat. Commun. 8, 249 (2017).

24. Zhang, Q. Y. et al. BTB-TAZ domain protein MdBT2 modulates malate accumulation and vacuolar acidification in response to nitrate. Plant Physiol. 183, 750-764 (2020)

25. Ren, S., Mandadi, K. K., Boedeker, A. L., Rathore, K. S. \& McKnight, T. D. Regulation of telomerase in Arabidopsis by BT2, an apparent target of TELOMERASE ACTIVATOR1. Plant Cell. 19, 23-31 (2007).

26. Figueroa, P. et al. Arabidopsis has two redundant Cullin3 proteins that are essential for embryo development and that interact with RBX1 and BTB proteins to form multisubunit E3 ubiquitin ligase complexes in vivo. Plant Cell. 17, 1180-1195 (2005).

27. Robert, H. S., Quint, A., Brand, D., Vivian-Smith, A. \& Offringa, R. BTB and TAZ domain scaffold proteins perform a crucial function in Arabidopsis development. Plant J. 58, 109-121 (2009).

28. Mandadi, K. K., Misra, A., Ren, S. \& McKnight, T. D. BT2, a BTB protein, mediates multiple responses to nutrients, stresses, and hormones in Arabidopsis. Plant Physiol. 150, 1930-1939 (2009).

29. An, J. P. et al. R2R3-MYB transcription factor MdMYB23 is involved in the cold tolerance and proanthocyanidin accumulation in apple. Plant J. 96, 562-577 (2018).

30. An, J. P. et al. EIN3-LIKE1, MYB1, and ETHYLENE RESPONSE FACTOR3 act in a regulatory loop that synergistically modulates ethylene biosynthesis and anthocyanin accumulation. Plant Physiol. 178, 808-823 (2018).

31. An, J. P., Wang, X. F. \& Hao, Y. J. BTB/TAZ protein MdBT2 integrates multiple hormonal and environmental signals to regulate anthocyanin biosynthesis in apple. Integr. Plant Biol. 0, 1-4 (2020).

32. Hu, D. G. et al. Glucose sensor MdHXK1 phosphorylates and stabilizes MdbHLH3 to promote anthocyanin biosynthesis in apple. PLoS Genet. 12, e1006273 (2016).
33. Ma, Q. J. et al. Transcription factor AREB2 is involved in soluble sugar accumulation by activating sugar transporter and amylase genes. Plant Physiol. 174, 2348-2362 (2017).

34. Hu, D. G. et al. The regulatory module MdPUB29-MdbHLH3 connects ethylene biosynthesis with fruit quality in apple. N. Phytol. 221, 1966-1982 (2019).

35. Chen, S., Songkumarn, P., Liu, J. \& Wang, G. L. A versatile zero background Tvector system for gene cloning and functional genomics. Plant Physiol. 150, 1111-1121 (2009).

36. Wang, X. F. et al. The nitrate-responsive protein MdBT2 regulates anthocyanin biosynthesis by interacting with the MdMYB1 transcription factor. Plant Physiol. 178, 890-906 (2018).

37. Xie, X. B. et al. The bHLH transcription factor MdbHLH3 promotesanthocyanin accumulation and fruit colouration inresponse to low temperature in apples. Plant Cell Environ. 35, 1884-1897 (2012).

38. Han, P. et al. BTB-BACK domain E3 ligase MdPOB1 suppresses plant pathogen defense against botryosphaeria dothidea by ubiquitinating and degrading MdPUB29 protein in apple. Plant Cell Physiol. 60, 2129-2140 (2019).

39. Zhao, Q. et al. Ubiquitination-related MdBT scaffold proteins target a bHLH transcription factor for iron homeostasis. Plant Physiol. 172, 1973-1988 (2016).

40. Sun, M. H. et al. The glucose sensor MdHXK1 phosphorylates a tonoplast $\mathrm{Na}^{+} / \mathrm{H}^{+}$Exchanger to improve salt tolerance. Plant Physiol. 176, 2977-2990 (2018).

41. Li, Y. Y. et al. MdCOP1 ubiquitin E3 ligases interact with MdMYB1 to regulate light-induced anthocyanin biosynthesis and red fruit coloration in apple. Plant Physiol. 160, 1011-1022 (2012).

42. Hu, D. G. et al. Overexpression of MdSOS2L1, an CIPK protein kinase, improves the antioxidant metabolites to enhance salt tolerance in apple and tomato. Physiol. Plant. 156, 201-214 (2016).

43. Lu, M., Vergara, S. \& Zhang, L. The amino-terminal domain of the E subunit of vacuolar $\mathrm{H}^{+}$-ATPase (V-ATPase) interacts with the $\mathrm{H}$ subunit and is required for V-ATPase function. J. Biol. Chem. 277, 38409-38415 (2002).

44. Wang, B., Luttge, U. \& Ratajczak, R. €. Effects of salt treatment and osmotic stress on V-ATPase and V-PPase in leaves of the halophyte Suaeda salsa. J. Exp. Bot. 52, 2355-2365 (2001).

45. Tang, R. J. et al. Tonoplast calcium sensors CBL2 and CBL3 control plant growth and ion homeostasis through regulating $\mathrm{V}$-ATPase activity in Arabidopsis. Cell Res. 22, 1650-1665 (2012).

46. Vierstra, R. D. The ubiquitin/26S proteasome pathway, the complex last chapter in the life of many plant proteins. Trends Plant Sci. 8, 135-142 (2003).

47. Adams, E. H. \& Spoel, S. H. The ubiquitin-proteasome system as a transcriptional regulator of plant immunity. J. Exp. Bot. 69, 4529-4537 (2018).

48. Shi, Y., Ding, Y. \& Yang, S. H. Molecular regulation of CBF signaling in cold acclimation. Trends Plant Sci. 23, 623-637 (2018).

49. An, J. P. et al. MdbHLH93, an apple activator regulating leaf senescence, is regulated by $\mathrm{ABA}$ and $\mathrm{MdBT} 2$ in antagonistic ways. N. Phytol. 222, 735-751 (2018). 